Entropy 2003, 5, 417-431

Entropy

ISSN 1099-4300

www.mdpi.org/entropy/

\title{
Droplet Size Distribution in Sprays Based on Maximization of Entropy Generation
}

\author{
Xianguo Li and Meishen Li \\ Department of Mechanical Engineering, \\ University of Waterloo, \\ Waterloo, Ontario, N2L 3G1 \\ Canada. \\ Tel: $1-519-888-4567$ ext. 6843 \\ Fax: 1-519-888-6197 \\ E-mail: x6li@uwaterloo.ca \\ http:// www.me.uwaterloo/ x6li
}

Received: 20 July 2003 / Accepted: 30 December 2003 / Published: 31December 2003.

\begin{abstract}
The maximum entropy principle (MEP), which has been popular in the modeling of droplet size and velocity distribution in sprays, is, strictly speaking, only applicable for isolated systems in thermodynamic equilibrium; whereas the spray formation processes are irreversible and non-isolated with interaction between the atomizing liquid and its surrounding gas medium. In this study, a new model for the droplet size distribution has been developed based on the thermodynamically consistent concept - the maximization of entropy generation during the liquid atomization process. The model prediction compares favorably with the experimentally measured size distribution for droplets, near the liquid bulk breakup region, produced by an air-blast annular nozzle and a practical gas turbine nozzle. Therefore, the present model can be used to predict the initial droplet size distribution in sprays.
\end{abstract}

Keywords: Sprays, droplet size distribution, entropy generation

\section{Introduction}

Atomization of liquids is a process used in a wide range of industrial operations. The surface area for a given amount of liquid can be increased considerably through this process. Consequently the processes associated with surface phenomena, e.g. the transport of mass and heat, can be enhanced greatly. For 
power generation systems, in addition to the above advantages, the pollutant emissions are also related to the quality of atomization. The distribution of droplet size and velocity in sprays has long been recognized as a crucial parameter needed for fundamental analysis of practical spray systems. Detailed information regarding droplet size and velocity distributions in sprays is of ultimate importance for the design, operation, and optimization of spray systems.

The empirical approach, finding a curve to best fit the measured data, is limited because the correlation constants lack universality and predictive capability for different sprays, and even for the same sprays under different flow conditions. Since the later 1980s, Maximum Entropy Principle (MEP) method has been applied popularly in the spray field to predict droplet size and velocity distribution and obtained reasonable success. The MEP approach can predict the most likely droplet size and velocity distributions under a set of constraints expressing the available information related to the distribution sought. The application of MEP to spray modeling was pioneered by Sellens and Brzustowski [1] and Li and Tankin [2]. When only first principles are used as constraints, the droplet size pdf does not go to zero in the formulation as the droplet size approaches zero. An additional constraint, partition of surface energy, is added by Sellens. Li solved this problem by defining a probability distribution as the number probability of finding a droplet in a spray within a volume bin. The number-based droplet size distribution in the resultant formulation goes to zero as the droplet size approaches zero. This is consistent with physical intuition and experimental observation. Other investigators have also implemented and developed this method in the past decade. Ahmadi and Sellens [3] reached a conclusion that prediction of the droplet size distribution is independent of the velocity distribution and the constraints on momentum and kinetic energy carry only velocity information. Cousin et al. [4] advocate a new approach in which the constraint is based on a single representative diameter instead of the commonly used conservation laws. Mitra and Li [5] combined a linear and nonlinear instability model with MEP method. The completely predictive model incorporates both the deterministic and stochastic aspect of spray droplet formation processes. However, MEP is not physically consistent with the real atomization process. Strictly speaking, MEP method is only applicable for isolated systems in thermodynamic equilibrium, whereas the spray formation process is non-isolated and irreversible. This leads to the difficulty of agreement between MEP-based distributions with various experimental data. The success of MEP method may be explained as the deviation from equilibrium condition is small for the cases investigated.

The major objective of the current study is to formulate a new model on the prediction of droplet size distribution based on the thermodynamically consistent concept - the maximization of entropy generation (MEG) during the spray formation which is actually a non-isolated and irreversible process. The entropy generation is analyzed based on the second thermodynamic law. Comparison between the model prediction and experimental data indicates good agreement between the two.

\section{Model Formulation}

Considering an atomizer, such as an air-blast atomizer, that produces flat or conical liquid sheets at the atomizer exit, the atomization process starts at the atomizer exit. As the liquid proceeds downstream, 
the thickness of the liquid sheet decreases; and instabilities set in, which break up the liquid sheet into ligaments and finally droplets. Therefore, the atomization ends at some downstream location where droplets are formed. Here the spray is assumed steady and isothermal. The control volume, as shown in Figure1, is taken from the atomizer exit to the droplet-formation plane.

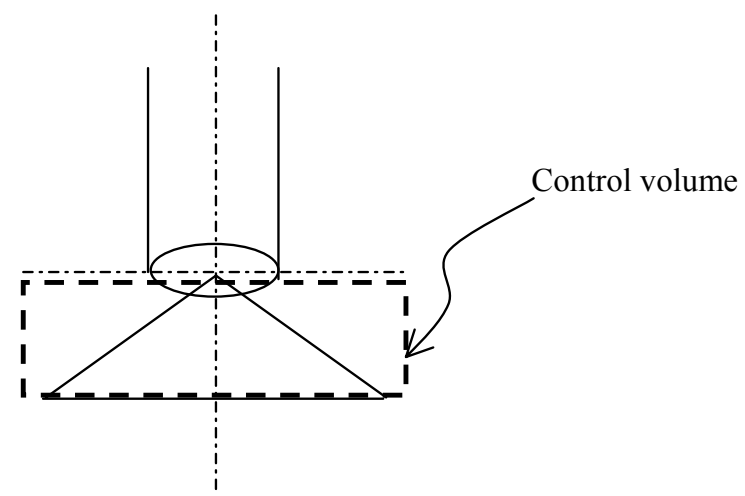

Figure 1. Schematic of the control volume chosen

Let $\dot{m}$ denote the mass flow rate at which the liquid is being sprayed, i.e., the amount of the liquid mass injected from the atomizer in a unit time, $\dot{N}_{\text {total }}$ the total number of droplets being produced per unit time and $P_{i}$ the number-based size probability distribution of droplets being produced at the plane of droplet formation. $P_{i}$ may be regarded as the number fraction of the droplets formed with volume $V_{i}$ or diameter $D_{i}$. The droplets may be assumed spherical due to the surface tension effect.

\subsection{Entropy analysis}

According to the second law in thermodynamics, we have,

$$
\left\{\begin{array}{c}
\text { entropy } \\
\text { increase } \\
\text { within C.V. . }
\end{array}\right\}=\left\{\begin{array}{c}
\text { entropy } \\
\text { flow } \\
\text { in }
\end{array}\right\}-\left\{\begin{array}{c}
\text { entropy } \\
\text { flow } \\
\text { out }
\end{array}\right\}+\left\{\begin{array}{c}
\text { entropy } \\
\text { generation }
\end{array}\right\}
$$

Or mathematically,

$\frac{d S_{c v}}{d t}=(\dot{m} \cdot s)_{1}-(\dot{m} \cdot s)_{2}+\dot{S}_{m} \cdot s_{\text {source }}+\dot{S}_{g e n}$

For the steady state steady flow process, the rate of entropy increase within the control volume vanishes. Here, the mass flow rates may be different between the two states (i.e., the state at the nozzle exit and at the droplet formation plane downstream) considering the possible mass exchange between the system and the surrounding (condensation or evaporation). The difference can be denoted as $\dot{S}_{m}=\dot{m}_{2}-\dot{m}_{1}$, which is commonly referred to as the mass source term, and the corresponding specific entropy is denoted as $s_{\text {source. }}$. 
Then Eq. (1) can be recast as

$\dot{S}_{\text {gen }}=\dot{S}_{2}-\dot{S}_{1}-\dot{S}_{m} \cdot s_{\text {source }}=\dot{m}_{2} \cdot s_{2}-\dot{m}_{1} \cdot s_{1}-\dot{S}_{m} \cdot s_{\text {source }}$

Or in the per unit mass flow rate form

$s_{\text {gen }}=\frac{\dot{S}_{g e n}}{\dot{m}_{1}}=\left(1+\bar{S}_{m}\right) \cdot s_{2}-s_{1}-\bar{S}_{m} \cdot S_{\text {source }}$

where $\bar{S}_{m}=\dot{S}_{m} / \dot{m}_{1}$ is the dimensionless source term.

At the nozzle exit, the flow at state 1 is in bulk liquid form with free surface produced. The entropy at the nozzle exit is due to the liquid bulk and the surface tension effect, hence, $\dot{S}_{1}=\dot{S}_{1(l)}=\dot{m}_{1} \cdot s_{1(l)}$. Since the control volume is around the liquid, $s_{\text {source }}=s_{(l)} \cong s_{1(l)}$ because the liquid is assumed isothermal. Eq. (3) can be rewritten as

$$
s_{g e n}=\left(1+\bar{S}_{m}\right) \cdot\left(s_{2}-s_{1(l)}\right)
$$

In the breakup region (state 2), a multitude of droplets forms and the total surface area is increased dramatically. Liquid phase exists inside each of the droplets. The total entropy is composed of two parts. One part is associated with the liquid bulk and is similar to that at the state 1; therefore may be denoted as $\dot{S}_{2(l)}=\dot{m}_{2} \cdot s_{2(l)}$. The other part is due to the existence of the numerous individual droplets, represented as $\dot{S}_{2(d)}$, which is directly associated with the particle nature of the droplets. Hence,

$\dot{S}_{2}=\dot{S}_{2(d)}+\dot{S}_{2(l)}=\dot{S}_{2(d)}+\dot{m}_{2} \cdot s_{2(l)}$

The formation of droplets is not deterministic but stochastic. The entropy at state 2 should be associated with the probability distributions of the droplet sizes. Different distribution results in different amount of entropy. To evaluate entropy quantitatively, an analogy between the present case and that of Gibbs ensemble in statistical thermodynamics may be made here. The entropy due to the droplet nature can be derived as, with details given elsewhere [6]

$\dot{S}_{2(d)}=-k_{B} \dot{N}_{\text {total }} \sum_{i} P_{i} \ln P_{i}$

where $P_{i}=\dot{n}_{i} / \dot{N}_{\text {total }}$ is the probability of finding droplets with diameter $D_{i}$ in the spray. $\dot{n}_{i}$ is the number of droplets produced per unit time and with diameter $D_{i}$.

Therefore, the rate of entropy generation in this irreversible atomization process can be expressed as

$\dot{S}_{g e n}=\dot{S}_{2(d)}+\left(\dot{m}_{2} \cdot s_{2(l)}-\dot{m}_{2} \cdot s_{1(l)}\right)=-k_{B} \dot{N}_{\text {total }} \sum_{i} P_{i} \ln P_{i}+\left(\dot{m}_{2} \cdot s_{2(l)}-\dot{m}_{2} \cdot s_{1(l)}\right)$

According to the Gibbs equation for a simple compressible substance with free interfaces, the liquid bulk entropy change can be expressed as 


$$
T d s_{(l)}=d u+p d v+\sigma d a
$$

where $a$ is the surface area of the free interface per unit mass, $\sigma$ is the surface tension. All other symbols have their usual meanings in thermodynamics, i.e. $T$ is temperature, $s_{(l)}$ is entropy, $u$ internal energy, $p$ pressure and $v$ specific volume. Since the atomization process is assumed isothermal, the internal energy, only a function of temperature, is unchanged. Then Eq. (8) becomes

$$
T d s_{(l)}=p d v+\sigma d a=-\kappa p v d p+\sigma d a
$$

where $\kappa=-\left(\frac{1}{v} \frac{d v}{d p}\right)_{T}$ is the isothermal compressibility of the liquid, a thermodynamic property whose dependence on pressure is negligible for liquids.

Rewriting Eq. (9) gives

$$
d s_{(l)}=-\frac{\kappa v}{T} p d p+\frac{\sigma}{T} d a
$$

Entropy is a thermodynamic property that depends only on the state of the system. Hence integrating Eq. (10) over the isothermal process results in,

$$
s_{2(l)}-s_{1(l)}=-\frac{\kappa v}{T} \frac{1}{2}\left(p_{2}^{2}-p_{1}^{2}\right)+\frac{\sigma}{T}\left(a_{2}-a_{1}\right)
$$

At the atomizer exit, a thin liquid sheet forms from the annular air-blast atomizer, and the liquid pressure is almost the same as the surrounding air pressure since the curvature effect is small and negligible. However, a circular liquid jet forms for solid-cone sprays produced by small orifice atomizers, the liquid pressure is larger than the ambient air pressure due to the surface tension effect. However, the specific value of $p_{1}$ will not affect the determination of the droplet size distribution as shown later. Therefore, for simplicity, we assume

$$
p_{1}=p_{g}
$$

For liquid pressure at state 2, let's consider only one liquid droplet. For a liquid droplet in the air, the pressure difference across the free interface is related to the surface tension effect as follows:

$$
p_{2}-p_{1}=p_{l}-p_{g}=\frac{4 \sigma}{D_{i}}
$$

Substituting Eq. (13) into Eq. (11) gives,

$$
s_{2(l), P_{i}}-s_{1(l)}=-\frac{\kappa v}{T}\left[\left(\frac{8 \sigma^{2}}{D_{i}^{2}}\right)+p_{1}\left(\frac{4 \sigma}{D_{i}}\right)\right]+\frac{\sigma}{T}\left(\frac{6}{\rho D_{i}}-a_{1}\right)
$$

At state 2, the total entropy flow rate for the liquid inside all the droplets is therefore 
$S_{2(l)}=\sum_{i} \dot{m}_{i} \cdot S_{2(l), P_{i}}=\sum_{i}\left\{-\rho \frac{\pi}{6} D_{i}^{3} \dot{n}_{i} \frac{\kappa v}{T}\left[\left(\frac{8 \sigma^{2}}{D_{i}^{2}}\right)+p_{1}\left(\frac{4 \sigma}{D_{i}}\right)\right]+\frac{\sigma}{T}\left(\dot{n}_{i} \pi D_{i}^{2}\right)\right\}-\frac{\sigma}{T} \dot{m}_{2} a_{1}+\dot{m}_{2} S_{1(l)}$

The entropy generation in the atomization process is therefore

$\dot{S}_{\text {gen }}=\left(-k_{B} \dot{N}_{\text {total }} \sum_{i} P_{i} \ln P_{i}\right)-\sum_{i} P_{i}\left[A D_{i}+B D_{i}^{2}\right]-\frac{\sigma}{T} \dot{m}_{2} a_{1}$

where $A=\frac{4 \pi}{3} \sigma^{2} \dot{N}_{\text {total }} \frac{\kappa}{T}$ and $B=-\frac{\sigma \dot{N}_{\text {total }} \pi}{T}\left(1-\frac{2}{3} p_{1} \kappa v\right)$

$A$ and $B$ are unknown constant for a given spray because $\dot{N}_{\text {total }}$ is often unknown, which could be determined by the relation $\dot{m}=\rho \frac{\pi}{6} D_{30}^{3} \dot{N}_{\text {total }}$. This equation may then be rewritten in the unit mass flow rate form as follows

$s_{\text {gen }}=-\left(k_{B} / \rho \frac{\pi}{6} D_{30}^{3}\right) \sum_{i} P_{i} \ln P_{i}-\sum_{i} P_{i}\left[E \bar{D}_{i}+F \bar{D}_{i}^{2}\right]-\frac{\sigma}{T}\left(1+\bar{S}_{m}\right) a_{1}$

where $\bar{D}_{i}=D_{i} / D_{30}, E=8 \sigma^{2} \frac{\kappa v}{T} / D_{30}^{2}$, and $F=-\frac{\sigma v}{T}\left(6-4 p_{1} \kappa\right) / D_{30}$.

\subsection{Droplet size distribution model}

At state 2, the droplet size distribution is in reality the initial distribution for droplets just formed in a spray. There are infinite sets of the probability distribution $P_{i}$ that can satisfy the global constraints on the atomization process such as the conservation laws. In accordance with irreversible thermodynamics, the least biased (or the most realistic) distribution is the one that maximizes the amount of entropy generated for the naturally occurring atomization process.

For the present study the constraints imposed on the atomization process are the conservation of liquid mass and the normalization condition. The normalization condition is

$\sum_{i} P_{i}=1$

stating the fact that the total probability for all the droplets in a spray should be equal to one.

The mass conservation under steady state requires that the sum of all droplets produced per unit time be equal to the mass of the liquid sprayed per unit time, plus the mass source term $\left(\dot{S}_{m}\right)$, which represents the rate of mass transfer between the liquid and the gas phase, i.e. the condensation or evaporation, during the atomization process. The expression for the conservation of mass can be written as:

$\sum_{i} \rho \frac{\pi}{6} D_{i}^{3} \dot{N}_{\text {total }} \cdot P_{i}=\dot{m}+\dot{S}_{m}$ 
Eq. (19) is nondimensionalized as,

$\sum_{i} P_{i} \bar{D}_{i}^{3}=1+\bar{S}_{m}$

where $\bar{S}_{m}=\dot{S}_{m} / \dot{m}$ denotes the dimensionless mass source term.

To maximize the entropy generation $s_{\text {gen }}$ under the constraints of Eq. (18) and Eq. (20), the Lagrange's method is adopted here. That yields,

$$
P_{i}=\exp \left(-\alpha_{0}-\alpha_{1} \bar{D}_{i}-\alpha_{2} \bar{D}_{i}^{2}-\alpha_{3} \bar{D}_{i}^{3}\right)
$$

where

$$
\begin{aligned}
& \alpha_{1}=\frac{E}{k_{B} / \rho \frac{\pi}{6} D_{30}^{3}}=\frac{4 \pi \sigma^{2} \kappa D_{30}}{3 k_{B} T} \\
& \alpha_{2}=\frac{F}{k_{B} / \rho \frac{\pi}{6} D_{30}^{3}}=-\frac{\pi \sigma D_{30}^{2}\left(6-4 p_{1} \kappa\right)}{6 k_{B} T}
\end{aligned}
$$

To obtain the probability of finding the droplets whose volume is between $\bar{V}_{n-1}$ and $\bar{V}_{n}$, we have to evaluate

$P\left\{\bar{V}_{n-1}<\bar{V}<\overline{V_{n}}\right\}=\sum_{\overline{V_{n-1}}}^{\overline{V_{n}}} P_{i}=\sum_{\overline{V_{n-1}}}^{\overline{V_{n}}} \exp \left(-\alpha_{0}-\alpha_{1} \bar{D}_{i}-\alpha_{2} \bar{D}_{i}^{2}-\alpha_{3} \bar{D}_{i}^{3}\right)$

It is generally regarded that the droplet size and velocity in sprays vary continuously rather than discretely. Therefore, the subscript $i$ can be dropped, and the summation form of Eq. (24) becomes an integral over the droplet size; that is

$P\left\{\bar{V}_{n-1}<\bar{V}<\bar{V}_{n}\right\}=\int_{\bar{V}_{n-1}}^{\bar{V}_{n}} \exp \left(-\alpha_{0}-\alpha_{1} \bar{D}-\alpha_{2} \bar{D}^{2}-\alpha_{3} \bar{D}^{3}\right) d \bar{V}$

The mean volume in a spray can be expressed in terms of mass mean diameter,

$V_{m}=\frac{\pi}{6} D_{30}^{3}$

Thus the non-dimensional droplet volume becomes

$$
\bar{V}=\frac{V}{V_{m}}=\left(\frac{D}{D_{30}}\right)^{3}=\bar{D}^{3}
$$


Substituting Eq. (27) into Eq. (25) leads to,

$$
P\left\{\bar{V}_{n-1}<\bar{V}<\bar{V}_{n}\right\}=P\left\{\bar{D}_{n-1}<\bar{D}<\bar{D}_{n}\right\}=\int_{\bar{D}_{n-1}}^{\bar{D}_{n}} f d \bar{D}=\int_{\bar{D}_{n-1}}^{\bar{D}_{n}} 3 \bar{D}^{2} \exp \left\{-\alpha_{0}-\alpha_{1} \bar{D}-\alpha_{2} \bar{D}^{2}-\alpha_{3} \bar{D}^{3}\right\} d \bar{D}
$$

where $\bar{D}_{n-1}$ and $\bar{D}_{n}$ are the droplet diameters corresponding to the droplet volume $\bar{V}_{n-1}$ and $\bar{V}_{n}$ respectively; and $f$ is the continuous droplet size probability density function (pdf). Thus,

$$
f=3 \bar{D}^{2} \exp \left\{-\alpha_{0}-\alpha_{1} \bar{D}-\alpha_{2} \bar{D}^{2}-\alpha_{3} \bar{D}^{3}\right\}
$$

The unknown Lagrangian multiplier $\alpha_{0}$ and $\alpha_{3}$ can be determined from the normalization and conservation of mass equations; and $\alpha_{1}$ and $\alpha_{2}$ from Eqs. (22) and (23) if $D_{30}$ is known. On the other hand, the above formulation is equivalent to the distribution from the MEP using an extended set of constraints. In addition to the normalization condition and the conservation of mass, two other constraints are written on the basis of the definition of the mean diameters $D_{10}$ and $D_{20}$. All the constraints that are needed to determine the unknown coefficients $\alpha_{i}$ 's are listed below.

$$
\begin{aligned}
& \int_{\bar{D}_{\text {min }}}^{\bar{D}_{\text {max }}} 3 \bar{D}^{2} \exp \left\{-\alpha_{0}-\alpha_{1} \bar{D}-\alpha_{2} \bar{D}^{2}-\alpha_{3} \bar{D}^{3}\right\} d \bar{D}=1 \\
& \int_{\bar{D}_{\text {min }}}^{\bar{D}_{\text {max }}} 3 \bar{D}^{3} \exp \left\{-\alpha_{0}-\alpha_{1} \bar{D}-\alpha_{2} \bar{D}^{2}-\alpha_{3} \bar{D}^{3}\right\} d \bar{D}=\bar{D}_{10} \\
& \int_{\bar{D}_{\text {min }}}^{\bar{D}_{\text {max }}} 3 \bar{D}^{4} \exp \left\{-\alpha_{0}-\alpha_{1} \bar{D}-\alpha_{2} \bar{D}^{2}-\alpha_{3} \bar{D}^{3}\right\} d \bar{D}=\bar{D}_{20}^{2} \\
& \int_{\bar{D}_{\text {min }}}^{\bar{D}_{\text {max }}} 3 \bar{D}^{5} \exp \left\{-\alpha_{0}-\alpha_{1} \bar{D}-\alpha_{2} \bar{D}^{2}-\alpha_{3} \bar{D}^{3}\right\} d \bar{D}=1+\bar{S}_{m}
\end{aligned}
$$

Here, it is worth noting that the model is only applicable to the immediate vicinity of the breakup region, where droplets are just formed in a spray.

\section{Results and Discussion}

Newton-Raphson method is used to solve the set of equations. The integrand over the diameter range involves exponential function. This makes Newton-Raphson method to be highly sensitive to the initial guess values of the parameters.

The experimental data are obtained from the measurements on an annular air-blast nozzle using a commercial phase Doppler Particle Analyzer (PDPA). The details of the measurement can be found in [7]. The nozzle is used to spray tap water exposed to inner and outer co-flowing air stream. It is assumed that the surrounding gas medium is fully saturated and therefore no mass transfer occurs between the liquid and the ambient gas from the nozzle exit to the breakup region. This assumption is reasonable for 
laboratory measurement of spray droplets near the atomizer exit because the enthalpy of vaporization is large and the vapour pressure of water is low at room temperature. Therefore the mass source term is set to zero. The water flow rate and the inner and outer airflow rates are adjusted in each case. The spatial distribution of droplet sizes and mean velocities and the mean diameters $D_{10}, D_{20}$ and $D_{30}$ are measured.

In the model, the control volume for the theoretical formulation is taken from the atomizer exit to the liquid sheet breakup region. This demands the measurement should be made as close to the breakup region as possible. Due to the difficulty encountered in measuring exactly at the breakup region, all the measurements are taken beyond that region. It is found that the discrepancy between model prediction and measurement data decreases with the reduction of the distance from the measurement location to the atomizer exit. In the available experimental data, the nearest position to the atomizer exit locates at $30 \mathrm{~mm}$ downstream. The specific flow conditions for each case are listed in Table 1.

\begin{tabular}{|c|c|c|c|}
\hline Case & $\begin{array}{c}\text { Water velocity } \\
(\mathrm{m} / \mathrm{s})\end{array}$ & $\begin{array}{c}\text { Inner air velocity } \\
(\mathrm{m} / \mathrm{s})\end{array}$ & $\begin{array}{c}\text { Outer air velocity } \\
(\mathrm{m} / \mathrm{s})\end{array}$ \\
\hline 1 & 2.1 & 48 & 48 \\
\hline 2 & 2.1 & 41 & 27 \\
\hline 3 & 3.2 & 27 & 41 \\
\hline 4 & 4.3 & 27 & 41 \\
\hline
\end{tabular}

Table 1. Flow conditions at the nozzle exit

Table 2 presents the corresponding arithmetic mean diameter $D_{10}$, the surface area mean diameter $D_{20}$ and mass mean diameter $D_{30}$ measured for each case listed in Table 1 along with the validation rate for the PDPA measurement.

\begin{tabular}{|l|l|l|l|l|}
\hline Case & $D_{10}(\mu \mathrm{m})$ & $D_{20}(\mu \mathrm{m})$ & $D_{30}(\mu \mathrm{m})$ & $\begin{array}{l}\text { Validation } \\
\text { Rate }(\%)\end{array}$ \\
\hline 1 & 112.8 & 135.4 & 161.3 & 61 \\
\hline 2 & 108.1 & 142.3 & 182.0 & 48 \\
\hline 3 & 131.6 & 163.3 & 197.5 & 61 \\
\hline 4 & 152.5 & 188.6 & 227.1 & 54 \\
\hline
\end{tabular}

Table 2. Experimentally measured parameters

The comparisons between the predicted and measured size probability distribution are illustrated in Fig. $2 \sim$ Fig. 5 . 


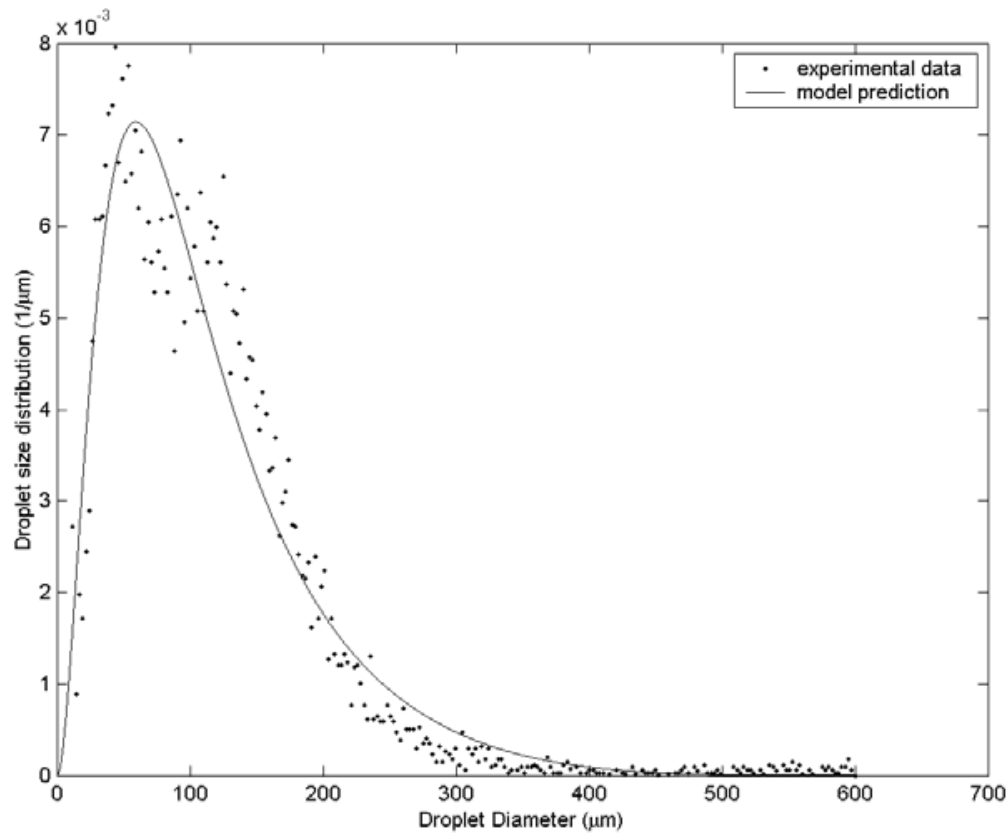

Figure 2. Comparison between the model prediction and measured droplet size probability distribution for Case 1

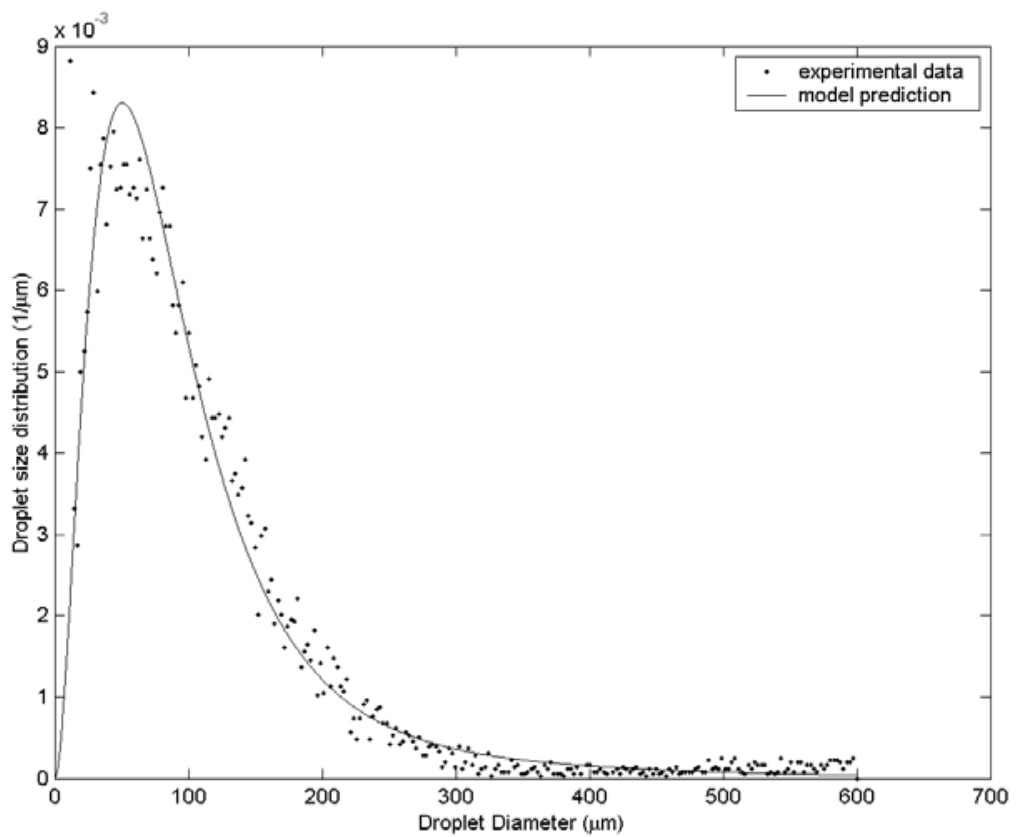

Figure 3. Comparison between the model prediction and measured droplet size probability distribution for Case 2 


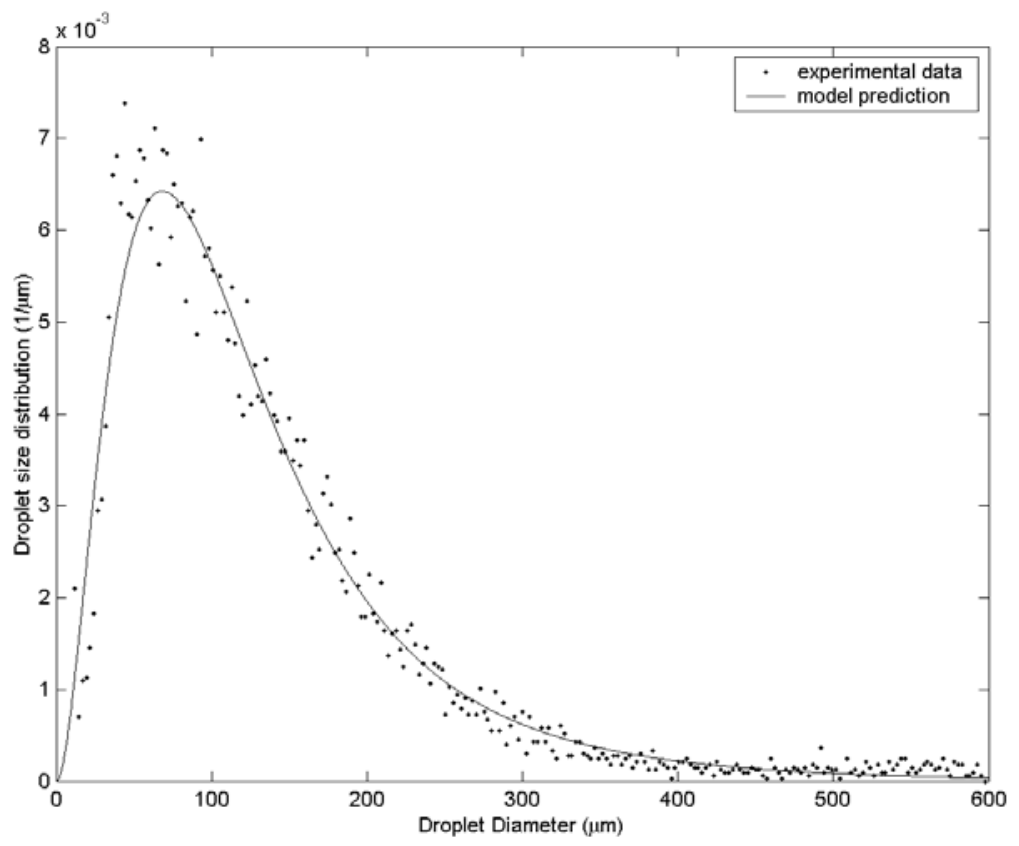

Figure 4. Comparison between the model prediction and measured droplet size probability distribution for Case 3

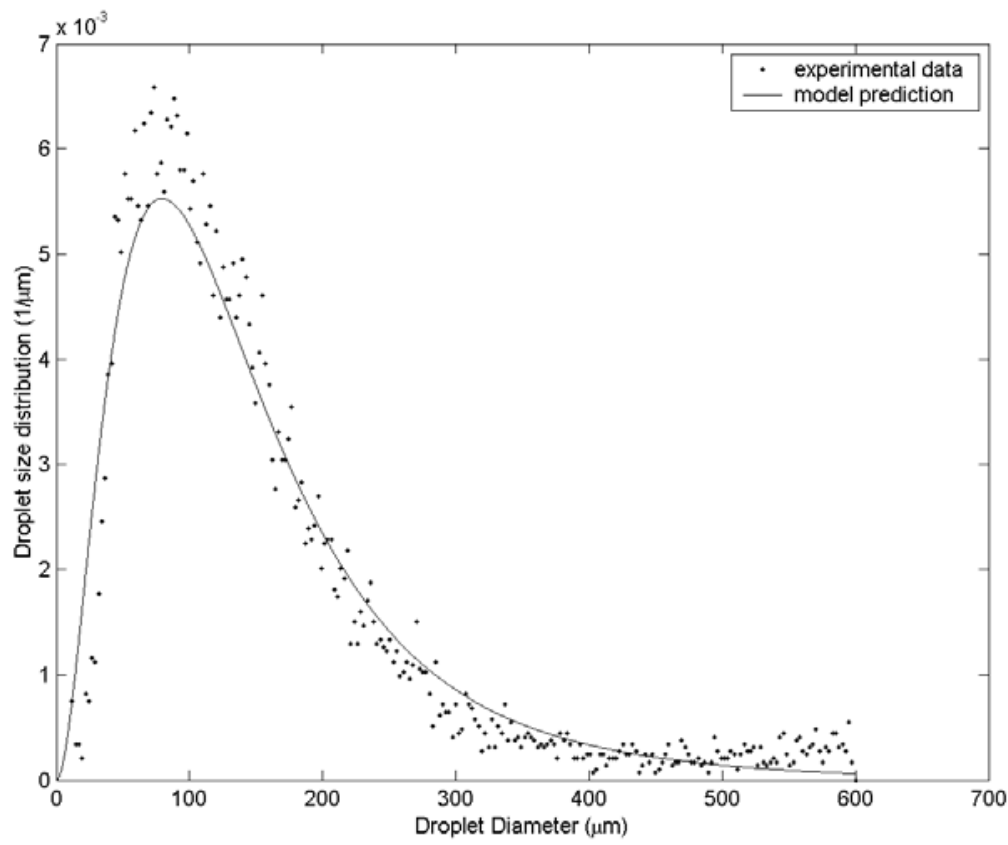

Figure 5. Comparison between the model prediction and measured droplet size probability distribution for Case 4 
Another comparison is made in this study with data measured for the actual gas turbine nozzle provided by Pratt \& Whitney Canada (PWC). Since this nozzle produces a good spray, a high validation rate is obtained, about $80 \%$ for the measured PDPA data. The details on the experimental measurement can be found in [8]. Four cases are demonstrated in the tables. Here, the case numbers are taken the same as those in [8]. The feature of the former distribution, eq. (29), is that the minimum diameter is always equal to zero. However, the measurement for the PWC nozzle indicates that the minimum diameter starts with a non-zero value. By introducing the minimum diameter $D_{0}$ into the equation as shown below, the distribution is modified to take this fact into account

$$
f=3\left(\bar{D}-\bar{D}_{0}\right)^{2} \exp \left\{-\alpha_{0}-\alpha_{1} \bar{D}-\alpha_{2} \bar{D}^{2}-\alpha_{3} \bar{D}^{3}\right\}
$$

The minimum diameter is taken from the experimental data and normalized with mass mean diameter. All the minimum diameters for the four cases are shown in Table 3. The unknown parameters, $\alpha_{i}{ }^{\prime} \mathrm{s}$, are still determined as before from the constraints equations (30) - (33).

\begin{tabular}{|l|l|l|l|l|}
\hline & Case I & Case II & Case III & Case IV \\
\hline$D_{0}(\mu \mathrm{m})$ & 12.385 & 16.321 & 14.350 & 13.697 \\
\hline
\end{tabular}

Table 3. Minimum diameters for PWC nozzle.

Fig. $6 \sim$ Fig. 9 show the comparisons between the present model prediction, eq. (34), and measured size probability distribution for the actual nozzle.

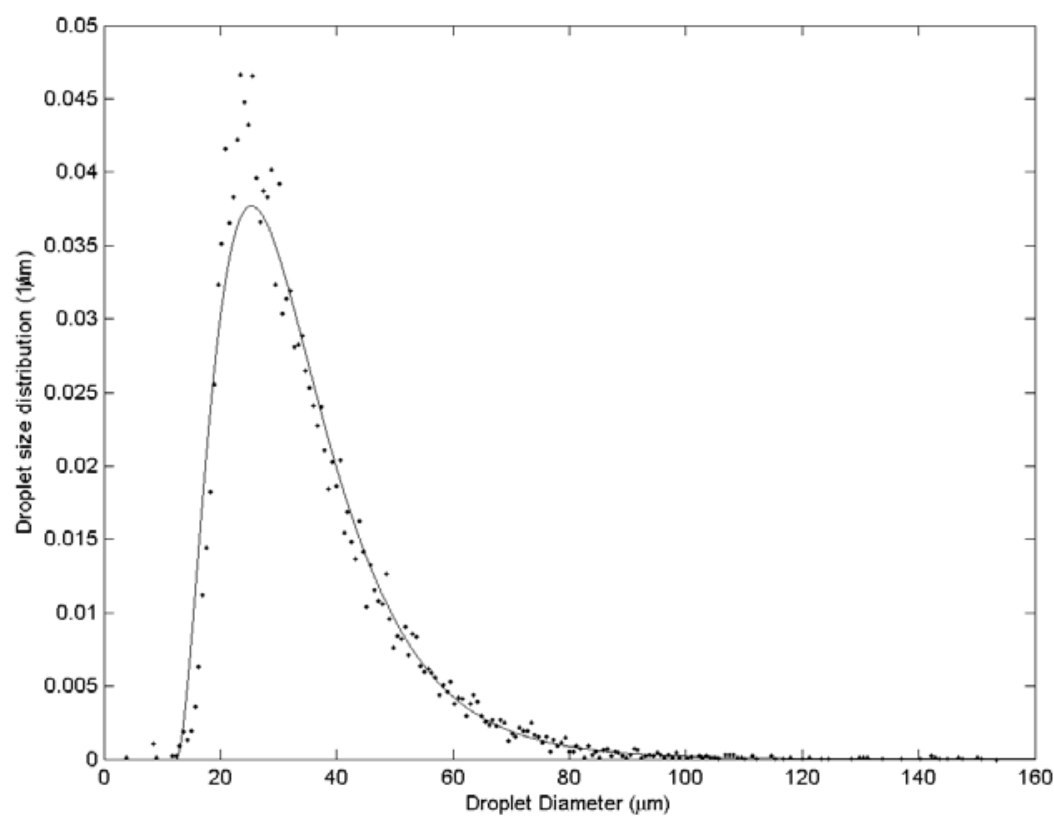

Figure 6. Comparison between the model prediction and measured droplet size probability distribution for the PWC nozzle (Case I) 


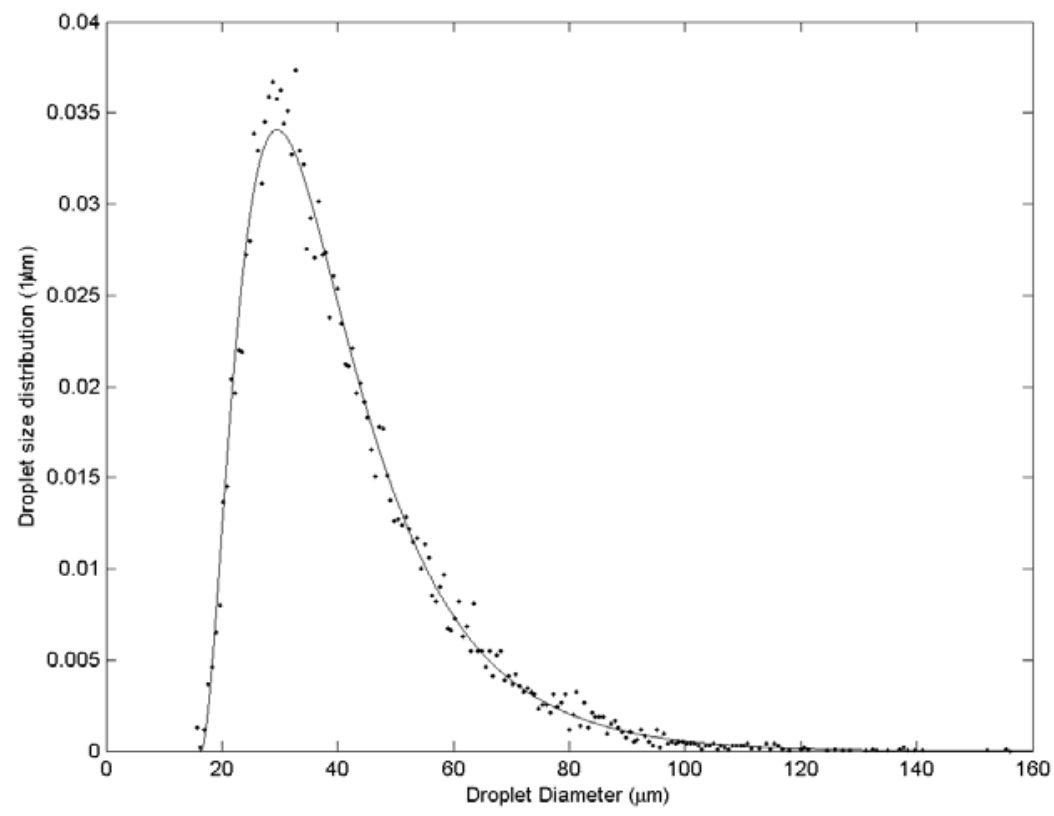

Figure 7. Comparison between the model prediction and measured droplet size probability distribution for the PWC nozzle (Case II)

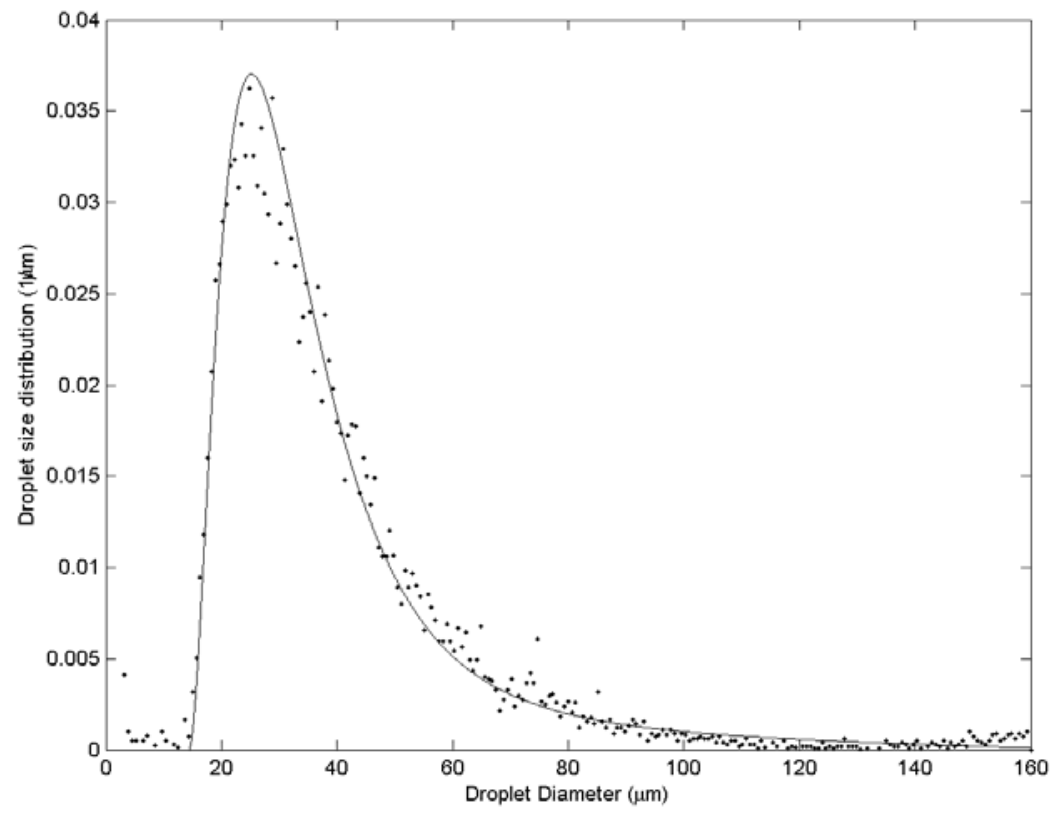

Figure 8. Comparison between the model prediction and measured droplet size probability distribution for the PWC nozzle (Case III) 


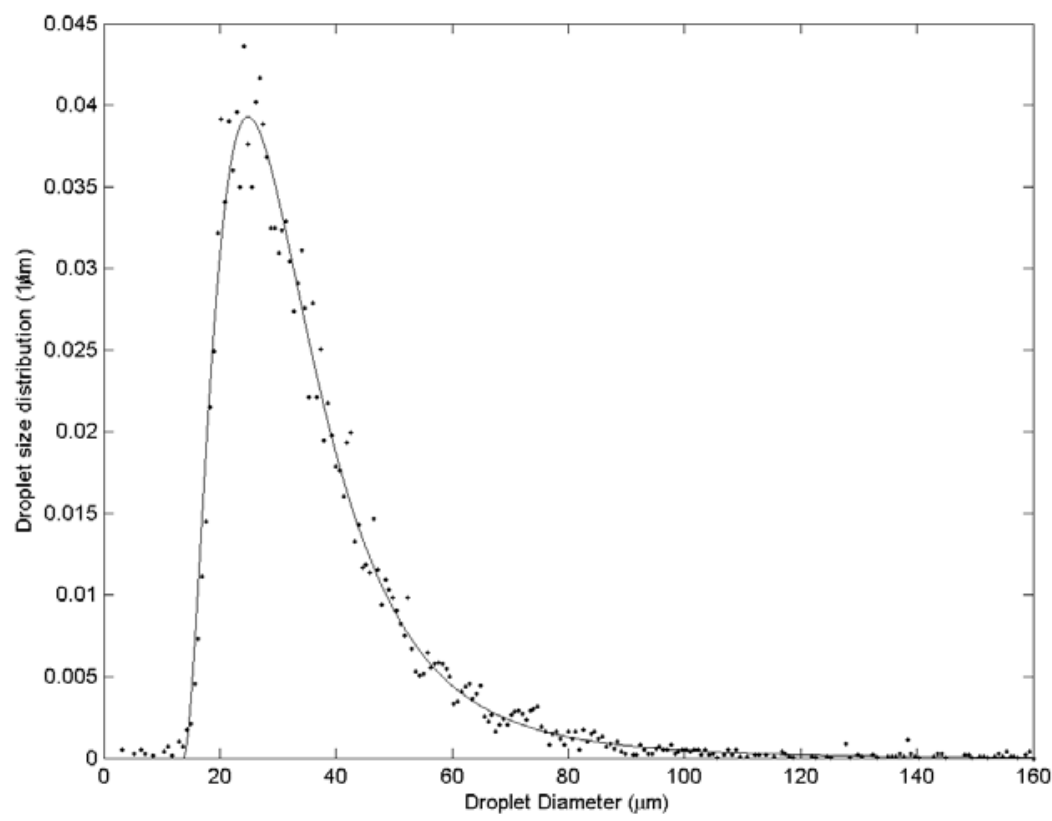

Figure 9. Comparison between the model prediction and measured droplet size probability distribution for the PWC nozzle (Case IV)

It is clear that the present model predictions agree well with the measured data for all four cases, and in fact, the agreement is much better than the complex approach based on the modified maximum entropy principle detailed in [8].

\section{Conclusion}

A new model for the droplet size distribution in sprays has been formulated based on the physically consistent concept - the maximization of entropy generation during the non-isolated and irreversible liquid atomization process. A comparison between the model prediction and experimentally measured droplet size distribution produced by an air-blast annular nozzle and a practical gas turbine nozzle indicates that the model prediction is in satisfactory agreement with the measurements. The present model can be used to predict the initial droplet size distribution in sprays. Further work is under way to extend the present model to the joint droplet size and velocity distribution.

\section{Acknowledgement}

The financial support of the Natural Sciences and Engineering Research Council of Canada (NSERC) is greatly appreciated. 


\section{References}

1. Sellens, R.W. and Brzustowski, T.A. (1985). A Prediction of the Drop Size and Velocity Distribution in a Spray from First Principles, Atomization and Spray Technology 1, 195-201

2. Li, X. and Tankin, R.S. (1988). Droplet Size Distribution: A Derivation of a Nukiyama-Tanasawa Type Distribution Function, Combustion Science and Technology 55, 65-76

3. Ahmadi, M. and Sellens, R.W. (1993). A Simplified Maximum-Entropy-Based Drop Size Distribution, Atomization and Sprays 3, 291-310

4. Cousin, J., Yoon, S.J. and Dumouchel, C. (1996). Coupling of Classical Linear Theory and Maximum Entropy Formalism for Prediction of Drop Size Distribution in Sprays: Application to Pressure-Swirl Atomizers, Atomization and Sprays 6, 601-622

5. Mitra, S.K. and Li, X. (1999). A Predictive Model for Droplet Size Distribution in Sprays, Atomization and Sprays 9, 29-50

6. Li, X., Li, M. and Fu, H., Modeling the Initial Droplet Size Distribution in Sprays Based on the Maximization of Entropy Generation, Atomization and Sprays, submitted

7. Fu, F. (2003). Experimental Characterization of Sprays from an Air-blast Annular Research Nozzle, Master's thesis, University of Waterloo

8. Kim, W.T., Mitra, S.K., Li, X., Prociw, L.A., Hu, T.C.J. (2003). A Predictive Model for the Initial Droplet Size and Velocity Distributions in Sprays and Comparison with Experiments, Part. Part. Syst. Charact. 20, 135-149

(C) 2003 by MDPI (http://www.mdpi.org). Reproduction for noncommercial purposes permitted. 